Susan Williams, MS, RD, MD, CCD, FACP, FACE

Endocrinology and Metabolism Institute, Cleveland Clinic,

Cleveland, $\mathrm{OH}$
Leila Khan, MD

Endocrinology and Metabolism Institute,

Cleveland Clinic, Cleveland, $\mathrm{OH}$
Angelo A. Licata, MD, PhD, FACP, FACE

Emeritus Consultant Staff, Endocrinology and Metabolism

Institute, Cleveland Clinic, Cleveland, $\mathrm{OH}$

\title{
DXA and clinical challenges of fracture risk assessment in primary care
}

\section{ABSTRACT}

Dual-energy x-ray absorptiometry (DXA) can detect bone mineral density loss before it can be identified on usual skeletal radiography, making it possible to diagnose osteoporosis in postmenopausal women and older men before clinical fractures arise. However, when DXA is used outside these populations or if the clinical picture does not match the reported T-scores, mistakes can arise in interpreting results and determining the need for pharmaceutical therapy.

\section{KEY POINTS}

While DXA is the gold standard test for measuring bone density, clinical judgment should take precedence if results contradict clinical information.

T-scores are not reliable indicators of fracture risk in premenopausal women, younger men, and children; Z-scores should be used for these populations.

Bone strength is now understood to depend on factors besides bone mineral density, sometimes causing discordance between DXA results and true fracture risk.

The Fracture Risk Assessment Tool incorporates clinical factors and can help guide treatment decisions.

New technologies directed at bone microarchitecture may one day improve risk analysis.
$\mathrm{O}$ UR UNDERSTANDING of dual-energy $x$-ray absorptiometry (DXA) is evolving as new information emerges about skeletal qualities that contribute to bone strength apart from bone mineral density (BMD). Some of these characteristics are not detectable by DXA analysis. Hence, overdependence on DXA results, particularly for patient populations that the test was not designed for, may lead to poor clinical decisions.

This article reviews the use of DXA and its limitations. Using case studies, clinical challenges of DXA scan interpretation are discussed, and guidance is provided in the diagnostic process and treatment decisions when clinical and DXA data are discordant.

\section{WHAT DXA DOES WELL}

DXA, originally developed for assessing fracture risk in postmenopausal women, ${ }^{1}$ is the gold standard test for diagnosing osteoporosis and monitoring its treatment. It can detect small but clinically relevant deficiencies in bone mass years before they are apparent on standard clinical radiographs, thereby allowing clinicians to intercede early to prevent fractures.

\section{T-scores}

DXA measures areal BMD (ie, bone mineral content divided by the bone scanned area) in the spine, hip, or forearm. Risk of fragility fracture is based on a calculated value called the T-score, which is the standard deviation of a patient's measurement from the mean of a young, healthy reference population. Values 
and their significance are as follows:

- -2.5 or below: highest fracture risk, diagnostic of osteoporosis and the need for pharmaceutic therapy

- -2.5 to -1.0: intermediate fracture risk, diagnostic of osteopenia (therapeutic approach may be uncertain)

- Above -1.0: lowest fracture risk, diagnosed as normal (usually no immediate concern for drug therapy).

As an assessment of fracture risk, T-scores are applicable only to untreated postmenopausal women and older men. Once drug therapy has started, T-scores do not accurately reflect risk.

With the widespread availability of DXA, physicians often use it to investigate skeletal concerns in populations other than postmenopausal women, including men, premenopausal women, children, teenagers, and young adults of both sexes. Such usage leads to challenges when interpreting DXA results.

Scientific advances have brought about a more complex understanding of the relationships between fracture risk, bone strength, and bone density. T-scores do not always correlate with fracture risk or even with a pa-
A T-score alone should not determine diagnosis and treatment tient's history of fracture and hence can be misinterpreted, leading to inappropriate treatment recommendations. A T-score should not solely determine diagnosis and treatment, and clinical data should appropriately modify the interpretation of results.

\section{THE DXA REPORT VS THE CLINICAL PRESENTATION: CASE SCENARIOS}

The following cases illustrate commonly encountered challenges if the DXA data and clinical presentation are incongruous.

\section{Case 1: A young runner with 'shin splints'}

A 35-year-old woman was referred for further guidance regarding a recent abnormal DXA test that had been ordered because of suspected "shin splints," ie, shin pain due to running. She was in good health, had been a recreational runner for decades with normal menstrual function, and had a healthy diet with no tobacco or alcohol use. She had no history of fractures but reported that a maternal relative may have had osteoporosis. She said that all women in her family were petite, had small stature, and had low bone density. Her examination showed small skeletal structure but was otherwise normal. Blood tests were normal. Review of the recent DXA report revealed Tscores of -0.6 for spine and -2.5 for hip. The report also included "borderline osteoporosis" in the hip and recommended drug therapy. The patient was psychologically traumatized by this information and sought further guidance.

\section{Case 2: A postmenopausal woman with intermediate T-scores}

A 60-year-old woman underwent her first DXA test, resulting in a T-score of -1.5 for spine and a score of -2.0 for the femoral neck. Her physician was pleased that the scores were not in the osteoporotic range, since she had undergone surgical menopause 30 years earlier. The physician's advice was to continue her healthy lifestyle, which included regular exercise, a vegan diet, and daily calcium and vitamin $D$ supplements. However, she had a radiologic and clinical diagnosis of spinal osteoarthritis, as well as bilateral wrist fractures from falls when she was in her 50s. Her family history included fractures in her mother and maternal grandmother. She used hormonal therapy after surgical menopause but stopped at age 45 due to concerns about risks and side effects. She has required no other prescription medication. Her blood test results were normal.

\section{The challenge of interpreting T-scores}

T-scores can be misinterpreted and lead to inappropriate treatment recommendations. Paradoxically, the patient in case 1 with a low hip T-score score may not be at immediate high risk of fracture, and a conservative approach maybe reasonable if there are no significant risk factors, while the patient in case 2 with the osteopenic T-score is at very high fracture risk and requires aggressive therapy.

What are the reasons for this paradox? Advances in clinical science have revealed more complexities in the concepts of fracture risk, bone strength, and bone density. A T-score alone should not be the final arbiter of diagnosis and treatment. Clinical data can modify the interpretation. The following discussion addresses the development of this concept, how it modified the notion of bone strength, DXA, and T-scores, and its clinical implications. 


\section{TABLE 1}

\section{Observations contributing to new understanding of bone density and bone strength}

Treatment with different antiresorptive drug classes led to similar vertebral fracture reduction despite different magnitudes of change in bone density. ${ }^{4,5}$

Early fracture rate improved with risedronate therapy despite no observable bone density changes. ${ }^{6}$

High and low doses of teriparatide led to similar rates of vertebral fracture reduction but different increases in bone density. ${ }^{7}$

Large-dose sodium fluoride to treat osteoporosis led to more fractures despite increased bone density. ${ }^{8}$

A high prevalence of low-impact fractures occurred despite abnormally elevated bone mineral density in 2 patients with autosomal-dominant osteopetrosis. ${ }^{9}$

Patients with diabetes have increased fracture risk despite normal bone density. ${ }^{10,11}$

Patients with hyperparathyroidism exhibit discordance between fracture rates and central and peripheral bone density. ${ }^{12}$

Fracture risk with glucocorticoids is independent of bone mineral density and correlates better with bone microarchitecture measures. ${ }^{13,14}$

More than half of older women with incident hip fracture did not have a diagnosis of osteoporosis up to 5 years previously. ${ }^{15}$

\section{BONE STRENGTH DEPENDS ON MORE THAN BONE MINERAL DENSITY}

DXA measures the amount of $x$-ray energy passing through bone and correlates it with the amount of mineral present. In theory, more mineral in bone (ie, greater density) indicates increased bone strength and fracture resistance, while less mineral indicates weaker bone that is more prone to fracture. Large population studies conducted in the development of DXA supported these correlations in postmenopausal women. ${ }^{1}$ This led to the Tscore system becoming the norm for diagnosing osteoporosis and high fracture risk and for determining the need for treatment.

But our understanding of the relationship between skeletal strength and bone density has evolved. Bone quality is now recognized to depend not only on density but also on skeletal characteristics that are not measured by DXA, including bone size and geometry, microarchitecture of trabecular and cortical compartments, cell turnover (reflecting metabolic activity), and composition of the mineralized protein matrix. ${ }^{2}$

\section{Drug trials reveal complexity}

Osteoporosis drugs produce a spectrum of changes in vertebral bone density. A 2019 meta-analysis ${ }^{3}$ that included 38 randomized drug trials and 19 antiresorptive and anabolic drugs found a strong correlation between improvements in BMD and greater reductions in rates of vertebral and hip fracture, reassuring practitioners of the usefulness of DXA to monitor treatment. However, drug effects on bone density explained only $48 \%$ to $63 \%$ of fracture reduction at the hip and spine.

Oddities have emerged in post hoc analyses of clinical trials that have led to new notions of bone strength (Table 1). ${ }^{4-15}$ In early pivotal trials, different drugs increased spinal BMD annually by different amounts while leading to similar incidences of clinical or radiologic vertebral fracture after 3 years of therapy: calcitonin (BMD increased 1.1\%; 33\% fracture reduction), risedronate (BMD increased 3.0$3.9 \%$; fracture reduction $41-49 \%)$, raloxifene (BMD increased 2.6\%; fracture reduction 30-50\%), and alendronate (BMD increased 3.2-5.7\%; fracture reduction 30-48\%). 4.5 In addition, some risedronate studies found that fracture reduction arose within 6 to 12 months of treatment without measurable changes in bone density, suggesting that other factors play a role. ${ }^{6}$
Microarchitecture has become a central tenet of the changing view of bone strength 


\section{TABLE 2}

\section{Clinical risk factors for fractures}

Older age

Low body weight and skeletal size

Family history of osteoporosis or fractures

Patient history of fractures

History of falls and imbalance

History of adult diseases compromising bone: endocrine disorders, bowel disease, nutritional disorders, renal disease

History of use of bone-toxic drugs: glucocorticoids, antiestrogens, antiandrogens, oncology agents

History of childhood disease impacting skeletal development

History of pubertal problems: delayed or absent puberty, amenorrhea, anorexia nervosa

History of harmful lifestyle: alcohol, tobacco, inactivity

Increased bone turnover markers

\section{Microarchitecture emerges} as a critical strength determinant

Microarchitecture has become a central tenet

Age is a major risk factor for fracture, independent of bone density of the changing view of bone strength. The 3-dimensional structure of interlocking bone plates, analogous to girders in buildings, confers intrinsic resistance to fracture. Increased osteoclastic and diminished osteoblastic activities in osteoporosis produce a degraded architectural network that is weakened and susceptible to fracture. ${ }^{16}$

\section{New measures of bone quality and strength}

Recent imaging technology has helped elucidate factors related to bone strength. Microarchitecture of bone can be visualized, and engineering protocols can be employed to measure its strength. High-resolution peripheral quantitative computed tomographic scanning is an important tool that produces 3-dimensional images of cortical and trabecular compartments in appendicular bone, with strength analyzed by finite element analysis. ${ }^{17-21}$ This technology, however, is generally limited to research centers.

The trabecular bone score uses a proprietary program to analyze information (ie, the gray-scale texture) in DXA images to gener- ate data about the integrity of the trabecular framework of vertebrae and, secondarily, fracture risk. High scores correlate with intact, nondegraded structure with low risk, and low scores correlate with degraded structure and high risk. Although available clinically, this program is not yet in widespread use. ${ }^{22,23}$

\section{INCORPORATING CLINICAL RISK INTO DXA INTERPRETATION}

DXA is unquestionably a useful tool to detect early bone loss, but results must be tempered with clinical judgment. Fractures can occur in a patient with any $\mathrm{T}$-score, ${ }^{24}$ analogous to occurrence of stroke with normal blood pressure and coronary events with normal lipid levels. The opportunity to prevent bone degradation may be missed if a practitioner waits for a high-risk patient's bone density to reach the T-score osteoporosis threshold. ${ }^{25}$

Because standard DXA analysis cannot detect microstructural change, clinicians must turn to other approaches to generate information about skeletal quality. In daily practice, the clinical history provides important data about risk factors, which in the broadest sense reveal information on microarchitecture (Table 2).

\section{Clinical risk calculators}

Most of the clinical risk factors are binary variables, and weighing their importance is subject to interpretation, often making a physician's experience the determining factor in estimating risk. Fracture risk calculators provide an objective numerical score to help guide decisions. ${ }^{26}$

Of the 13 risk calculators in use, the Fracture Risk Assessment Tool (FRAX), Garvan, and QFracture have been studied extensively. They are especially useful when access to DXA is limited. They vary in the number of variables used in their analyses. Their use may be restricted to specific geographic populations, and they do not quantify factors in the calculations such as duration and amount of glucocorticoid use and the severity of secondary diseases. They are intended for older people and have limited applicability to young patients. They may underestimate actual risk. 


\section{Age is a critical risk factor}

More than 3 decades ago, a seminal study found that age is a major risk factor for fracture, independent of bone density. ${ }^{27}$ With aging, fracture rates rise exponentially as bone density decreases. Fractures are less likely to occur in younger people than in older people, even with similar bone density measurements. ${ }^{28}$ Clinical data show that this paradox reflects age-dependent microarchitecture degradation. As a result, a young patient with low bone density may not be at high risk for fracture unless other clinical factors are present, and an older patient with nonosteoporotic T-scores could be at high risk for fracture because of other clinical risk factors.

Age is a variable in all clinical risk calculators. FRAX uses age to generate intervention thresholds for fracture with or without measured bone density.

\section{FRAX: The most important risk calculator}

FRAX has worldwide applicability and validation in different countries. ${ }^{29}$ Its calculations may be part of a DXA report, or clinicians may use web-based tools to run the calculations. It provides an intervention threshold for decision analysis.

Although FRAX is used worldwide, studies suggest that its thresholds may not be universal but ideally should be generated based on the specific geographic population of the patient. ${ }^{29}$ Many countries use the US National Osteoporosis Foundation guidelines, specifying that drug therapy be initiated if hip fracture risk is at least $3 \%$ or major osteoporotic fracture risk at least 20\%.

FRAX can provide fracture risk assessment from age alone but is more precise if hip BMD is added, with or without associated risk factors such as previous fracture, parental hip fracture, current smoking status, glucocorticoid use, rheumatoid arthritis, secondary osteoporosis, and alcohol use ( $\geq 3$ units per day). These are dichotomous variables in the calculator, but practitioners often consider quantitative aspects (eg, amount and duration of glucocorticoid use, severity and type of secondary osteoporosis) in their assessment and decision.

FRAX now allows the use of trabecular bone score data to help calculate intervention thresholds for major and hip osteoporotic frac- tures. It significantly improves risk prediction in patients with otherwise borderline FRAX results. ${ }^{30}$

\section{BONE TURNOVER MARKERS ADD INFORMATION}

For clinical use, the International Osteoporosis Foundation proposed the $\mathrm{C}$-telopeptide of type 1 collagen (CTX) as a biochemical marker of bone resorption and $\mathrm{N}$-propeptide of type 1 procollagen (P1NP) as a marker of bone formation. ${ }^{31,32}$ These are not diagnostic tools for osteoporosis and are not a substitute for DXA analysis.

These markers reflect bone metabolism or turnover. During menopause and in untreated osteoporosis, bone markers can be increased and indicate high skeletal turnover. Based on test results, menopausal women can be grouped as fast or slow "bone-losers." Cohort studies show bone loss is greater and fracture risk is higher as these biomarkers increase. However, for an individual patient, it is difficult to quantify this relationship, and the markers do not accurately predict bone loss or its magnitude.

But in clinical practice, these markers can help monitor patient adherence and drug efficacy $^{33}$ : antiresorptive drugs reduce levels of CTX and P1NP. The least significant change (ie, the smallest difference between successive measurements likely to be real change rather than chance) varies with the type of assay used. Depending on the assay, the least significant change in CTX is $50 \%$ to $54 \%$, and the least significant change in P1NP is $23 \%$ to $29 \%$. The expected clinical response is a $74 \%$ to $75 \%$ reduction in CTX and a $51 \%$ to $54 \%$ reduction in P1NP. ${ }^{31}$ Individual measurement variability occurs from circadian rhythms, meal patterns, and laboratory techniques. Consistency in sample acquisition (eg, early morning, fasting specimens) and use of the same testing laboratory help minimize variability. ${ }^{34}$

\section{Z-SCORES FOR YOUNGER PATIENTS}

The Z-score, calculated as standard deviations from the mean of a reference group matched by age, ethnicity, and sex, should be used instead of the T-score when assessing fracture
Fracture risk calculators provide an objective numerical score to help guide decisions 
risk in children, premenopausal women, and men younger than age $50 .{ }^{35}$ Some advocate its use in older patients in addition to the $T$ score.

The Z-score is infrequently seen in dictated patient reports but can be found in the scan images of the DXA test. A low value, ie, less than -2.0 , signals a lower bone mass than predicted and should prompt further investigation if the clinical history warrants.

\section{CASE 1 REVISITED}

The 35-year-old runner with a diagnosis of osteoporosis based on a low DXA hip T-score exemplifies a cascade of errors in fracture risk assessment. She may actually not be at immediate high risk of fracture, and a conservative approach may be reasonable.

The initial mistake was to order DXA for shin splints, which is not an indication for the test. This led to inappropriate use of Tscores, an incorrect diagnosis, prescription of a bisphosphonate in a healthy premenopausal woman with low fracture risk (and a chance of pregnancy), and unnecessary psychological turmoil for the patient.

Clinical factors are paramount in this case. The patient's normal menstrual function implies sufficient estrogen production that should protect her skeleton without requiring additional medication. However, a low bone density may be suspicious for secondary problems and warrants a thorough family and clinical history to reveal possible causes. Laboratory testing would corroborate treatable options. A conundrum arises when no firm diagnosis can be found and the patient is healthy.

A diagnosis of borderline osteoporosis is contrary to guidelines of the International Society for Clinical Densitometry, ${ }^{36}$ and "low bone mass for age" is the preferred diagnostic term. Assessment with Z-scores rather than Tscores is appropriate for this healthy premenopausal woman. It is unclear whether her low bone density represents a loss of bone from a higher baseline, which may represent an underlying disease state, or whether she may have a genetic phenotype of low density despite having strong bone. ${ }^{37}$ Her family history and clinical examination are notable for small skeletal structure, suggestive of genetic inheri- tance of low bone mass. Studies of healthy premenopausal women with low bone mass show a spectrum of microarchitectural changes that mimic, to a minor degree, changes similar to osteoporosis. However, these women have a low risk of fracture. It is speculated that such architectural changes represent a pre-osteoporosis state. ${ }^{38}$

\section{Management involves monitoring}

A T-score indicating low bone density should not be ignored. Striving to maintain bone mass should be the guiding management principle, as she will enter menopause with a low bone mass and may experience fractures from estrogen deficiency earlier than expected for her age. A healthy lifestyle with adequate exercise and diet is the minimal therapeutic strategy. Attention should also be directed to any problems in menstrual function, eating, and hormonal disorders that may arise that would accelerate bone loss. However, skeletal pharmaceutical agents should not be considered unless evidence of bone fragility develops.

Surveillance of bone density with DXA is warranted, but a recommended interval has not been established in a premenopausal patient this young. In our practice, we consider every 5 to 10 years to be reasonable if no skeletal problems or illnesses arise.

\section{CASE 2 REVISITED}

In the case of the 60-year-old woman, the bone density report was misleading and discordant with the history of wrist fractures. Although vertebral and hip fractures attract the most attention from clinicians, wrist fractures also occur frequently and, unfortunately, are less likely to raise concern for osteoporosis evaluation and treatment. Data from a Medicare cohort showed only $7 \%$ of patients with wrist fractures have DXA testing within 6 months of such injuries, yet 20\% later develop fractures of the hip or spine. ${ }^{39}$

The American Association of Clinical Endocrinologists and the American College of Endocrinology point out that errors in DXA scan acquisition and analysis can affect interpretation, and they encourage clinicians to review actual scan images and data rather than rely solely on a report, especially when 
it is discordant with the clinical picture. ${ }^{40} \mathrm{As}$ suming this patient's DXA report was of good quality, there are other reasons to question whether it reflects actual bone risk. Spinal arthritis can cause spinal DXA to be falsely normal and obscure bone deficiency. ${ }^{41,42}$ In women with a history of fracture, trabecular bone score technology usually reveals abnormal bone even when T scores are normal. ${ }^{43}$

\section{Pharmaceutical management recommended}

This patient's multiple risk factors (ie, age, history of fractures, and estrogen deficiency since stopping hormone replacement after hysterectomy) attest to a weakened skeleton from osteoporosis, and a T-score that meets the osteoporosis threshold is not required to begin pharmaceutical treatment.

Further, advising only the use of calcium and vitamin $\mathrm{D}$ is inadequate management. Her provider should recommend that she use an antiresorption agent as first-line therapy and consider anabolic drugs if there are problems with the initial drug choice. She should not reinstate hormone therapy at her age for bone health alone as there may be increased risk for cardiovascular disease. ${ }^{44}$ However, this caveat is not absolute and requires a balance of risk and reward if hormone therapy is also needed for vasomotor, genitourinary, or other problems.

\section{RECOMMENDATIONS FOR ASSESSING FRACTURE RISK}

Clinicians can expect to see patients similar to those in these 2 cases. Population data indicate that $2.5 \%$ of premenopausal women

\section{REFERENCES}

1. WHO Scientific Group on the Prevention and Management of Osteoporosis. Prevention and management of osteoporosis: report of a WHO scientific group. World Health Organization. https://apps. who.int/iris/handle/10665/42841. Accessed October 18, 2021.

2. Choksi P, Jepsen KJ, Clines GA. The challenges of diagnosing osteoporosis and the limitations of currently available tools. Clin Diabetes Endocrinol 2018; 4:12. doi:10.1186/s40842-018-0062-7

3. Bouxsein ML, Eastell R, Lui LY, et al; FNIH Bone Quality Project. Change in bone density and reduction in fracture risk: a metaregression of published trials. J Bone Miner Res 2019; 34(4):632-642. doi:10.1002/jbmr.3641

4. Wasnich RD, Miller PD. Antifracture efficacy of antiresoprtive agents are related to changes in bone density. J Clin Endocrinol Metab 2000; 85(1):231-236. doi:10.1210/jcem.85.1.6267

5. Delmas PD, Li Z, Cooper C. Relationship between changes in bone mineral density and fracture risk reduction with antiresorptive drugs: some issues with meta-analyses. J Bone Miner Res 2004; 19(2):330-337. doi:10.1359/JBMR.0301228

6. Roux C, Seeman E, Eastell R, et al. Efficacy of risedronate on clinical have T-scores below the reference range, and $6 \%$ of patients with any fracture have normal BMD tests. ${ }^{45}$ We recommend the following approach.

\section{Assess patients using DXA and FRAX}

These are still the major tools for assessing fracture risk. However, their results should not be regarded as absolute. The practitioner, not the technology, is the final arbiter for diagnosing disease.

\section{Use T-scores as a guide for postmenopausal women and older men}

A T-score of less than -2.5 is the intervention threshold for diagnosing and treating osteoporosis. However, keep in mind that patients with spine or hip T-scores in the normal or osteopenic range may require treatment for osteoporosis if the clinical history shows fractures. In such cases, one should not wait for T-scores to reach the critical -2.5 before intervening.

Use Z-scores for premenopausal women and young patients of both sexes. A low bone $\mathrm{Z}$-score in healthy men or women indicates a generally low risk for fracture and is adequately treated with good nutrition, exercise, healthy lifestyle, and skeletal surveillance. In the presence of fractures, illness that might affect the skeleton, or other risk factors, a more aggressive therapeutic approach may be indicated.

\section{DISCLOSURES}

The authors report no relevant financial relationships which, in the context of their contributions, could be perceived as a potential conflict of interest. 
doi:10.1530/EC-18-0456

12. Marcocci C, Cianferotti L, Cetani F. Bone disease in primary hyperparathyroidism. Ther Adv Musculosket Dis 2012; 4(5):357-368. doi:10.1177/1759720X12441869

13. Kanis JA, Johansson $\mathbf{H}$, Oden A, et al. A meta-analysis of prior corticosteroid use and fracture risk. J Bone Miner Res 2009; 19(6):893899. doi:10.1359/JBMR.040134

14. Graeff $\mathbf{C}$, Marin F, Petto $\mathbf{H}$, et al. High resolution quantitative computed tomography-based assessment of trabecular microstructure and strength estimates by finite-element analysis of the spine, but not DXA, reflects vertebral fracture status in men with glucocorticoid-induced osteoporosis. Bone 2013; 52(2):568-577. doi:10.1016/j.bone.2012.10.036

15. Wainwright SA, Marshall LM, Ensrud KE, et al. Hip fracture in women without osteoporosis. J Clin Endocrinol Metab 2005; 90(5):2787-2793. doi:101210/jc.2004-1568

16. Hunt HB, Donnelly E. Bone quality assessment techniques: geometric, compositional, and mechanical characterization from macroscale to nanoscale. Clin Rev Bone Miner Metab 2016; 14(3):133-149. doi:10.1007/s12018-016-9222-4.

17. Burghardt AJ, Issever AS, Schwartz AV, et al. High-resolution peripheral quantitative computed tomographic imaging of cortical and trabecular bone microarchitecture in patients with type 2 diabetes mellitus. J Clin Endocrinol Metab 2010; 95(11):5045-5055. doi:10.1210/jc.2010-0226

18. Cusano NE, Rubin MR, Silva BC, et al. Skeletal microstructure and estimated bone strength improve following parathyroidectomy in primary hyperparathyroidism. J Clin Endocrinol Metab 2018; 103(1):196-205. doi:10.1210/jc.2017-01932

19. Engelke $K$, van Rietbergen $B$, Zysset P. FEA to measure bone strength: a review. Clinic Rev Bone Miner Metab 2016; 14:26-37. doi:10.1007/s12018-015-9201-1

20. Vilayphiou N, Boutroy $\mathbf{S}$, Szulc $\mathbf{P}$, et al. Finite element analysis performed on radius and tibia HR-pQCT images and fragility fractures at all sites in men. J Bone Miner Res 2011; 26(5):965-973. doi:10.1002/jbmr.297

21. Tsai JN, Uihlein AV, Burnett-Bowie SA, et al. Comparative effects of teriparatide, denosumab, and combination therapy on peripheral compartmental bone density, microarchitecture, and estimated strength: the DATA-HRpQCT Study. J Bone Miner Res 2015; 30(1):3945. doi:10.1002/jbmr.2315

22. Silva BC, Leslie WD, Resch H, et al. Trabecular bone score: a noninvasive analytical method based upon the DXA image. J Bone Miner Res 2014; 29(3):518-530. doi:10.1002/jbmr.2176

23. Silva BC, Broy SB, Boutroy S, Schousboe JT, Shepherd JA, Leslie WD Fracture risk prediction by non-BMD DXA measures: the 2015 ISCD official positions part 2: trabecular bone score. J Clin Densitom 2015; 18(3):309-330. doi:10.1016/j.jocd.2015.06.008

24. Siris ES, Miller PD, Barrett-Connor E, et al. Identification and fracture outcomes of undiagnosed low bone mineral density in postmenopausal women: results from the National Osteoporosis Risk Assessment. JAMA 2001; 286(22):2815-2822. doi:10.1001/jama.286.22.2815

25. Leslie WD, Seeman E, Morin SN, Lix LM, Majumdar SR. The diagnostic threshold for osteoporosis impedes fracture prevention in women at high risk for fracture: a registry-based cohort study. Bone 2018; 114:298-303. doi:10.1016/j.bone.2018.07.004

26. Kanis JA, Johansson H, Harvey NC, McCloskey EV. A brief history of FRAX. Arch Osteoporos 2018; 3(1):118. doi:10.1007/s11657-018-0510-0

27. Hui SL, Slemenda CW, Johnston CC Jr. Age and bone mass as predictors of fracture in a prospective study. J Clin Invest 1988; 81(6):18041809. doi:10.1172/JCI113523

28. Marques A, Ferreira RJ, Santos E, Loza E, Carmona L, da Silva JA. The accuracy of osteoporotic fracture risk prediction tools: a systematic review and meta-analysis. Ann Rheum Dis 2015; 74(11):19581967. doi:10.1136/annrheumdis-2015-207907

29. Kanis JA, Harvey NC, Cooper C, et al. A systematic review of intervention thresholds based on FRAX: a report prepared for the
National Osteoporosis Guideline Group and the International Osteoporosis Foundation. Arch Osteoporos 2016; 11(1):25. doi:10.1007/s11657-016-0278-z

30. Martineau P, Leslie WD, Johansson $\mathbf{H}$, et al. Clinical utility of using lumbar spine trabecular bone score to adjust fracture probability: the Manitoba BMD cohort. J Bone Miner Res 2017; 32(7):1568-1574. doi:10.1002/jbmr.3124

31. Eastell R, Pigott T, Gossiel F, Naylor KE, Walsh JS, Peel NFA. Diagnosis of endocrine disease: bone turnover markers: are they clinically useful? Eur J Endocrinol 2018; 178(1):R19-R31. doi:10.1530/EJE-17-0585

32. Wheater G, Elshahaly M, Tuck SP, Datta HK, van Laar JM. The clinical utility of bone marker measurements in osteoporosis. J Transl Med 2013; 11:201. doi:10.1186/1479-5876-11-201

33. Eastell R, Black DM, Lui LY, et al. Treatment-related changes in bone turnover and fracture risk reduction in clinical trials of antiresorptive drugs: proportion of treatment effect explained. J Bone Miner Res 2021; 36(2):236-243. doi:10.1002/jbmr.4178

34. Vasikaran $\mathbf{S}$, Eastell $\mathbf{R}$, Bruyère $\mathbf{O}$, et al. Markers of bone turnover for the prediction of fracture risk and monitoring of osteoporosis treatment: a need for international reference standards. Osteoporos Int 2011; 22(2):391-420. doi:10.1007/s00198-010-1501-1

35. Carey JJ, Delaney MF. T-scores and Z-scores. Clinic Rev Bone Miner Metab 2010; 8:113-121. doi:10.1007/s12018-009-9064-4

36. Writing Group for the ISCD Position Development Conference. Diagnosis of osteoporosis in men, premenopausal women, and children. J Clin Densitom 2004; 7(1):17-26. doi:10.1385/jcd:7:1:17

37. Galusca B, Zouch M, Germain N, et al. Constitutional thinness: unusual human phenotype of low bone quality. J Clin Endocrinol Metab 2008; 93(1):110-117. doi:10.1210/jc.2007-1591

38. Cohen A, Dempster DW, Recker RR, et al. Abnormal bone microarchitecture and evidence of osteoblast dysfunction in premenopausal women with idiopathic osteoporosis. J Clin Endocrinol Metab 2011; 96(10):3095-3105. doi:10.1210/jc.2011-1387

39. Parikh K, Reinhardt D, Templeton K, Toby B, Brubacher J. Rate of bone mineral density testing and subsequent fracture-free interval after distal forearm fracture in the Medicare population. J Hand Surg Am 2021; 46(4):267-277. doi:10.1016/j.jhsa.2020.11.020

40. Licata AA, Binkley N, Petak SM, Camacho PM. Consensus statement by the American Association of Clinical Endocrinologists and American College of Endocrinology on the quality of DXA scans and reports. Endocr Pract 2018; 24(2):220-229. doi:10.4158/CS-2017-0081

41. Yu W, Glüer CC, Fuerst T, et al. Influence of degenerative joint disease on spinal bone mineral measurements in postmenopausal women. Calcif Tissue Int 1995; 57(3):169-174. doi:10.1007/BF00310253

42. Liu G, Peacock M, Eilam O, Dorulla G, Braunstein E, Johnston CC. Effect of osteoarthritis in the lumbar spine and hip on bone mineral density and diagnosis of osteoporosis in elderly men and women. Osteoporos Int 1997; 7(6):564-569. doi:10.1007/BF02652563

43. Binkley N, Morin SN, Martineau P, Lix LM, Hans D, Leslie WD. Frequency of normal bone measurement in postmenopausal women with fracture: a registry-based cohort study. Osteoporos Int 2020; 31(12):2337-2344. doi:10.1007/s00198-020-05576-w

44. Flores VA, Pal L, Manson JE. Recommended hormone therapy in menopause: concepts, controversies and approach to treatment [published online ahead of print, 2021 Apr 15]. Endocr Rev 2021; bnab011. doi:10.1210/endrev/bnab011

45. Lewiecki EM. Premenopausal bone health assessment. Curr Rheumatol Rep 2005; 7(1):46-52. doi:10.1007/s11926-005-0008-9

Address: Angelo A. Licata, MD, PhD, Department of Endocrinology, Diabetes, and Metabolism, F20, Cleveland Clinic, 9500 Euclid Avenue, Cleveland, OH 44195; licataa@ccf.org 


\section{DXA and fracture risk assessment}

In the November 2021 issue, an error appeared in Williams S, Khan L, Licata AA. DXA and clinical challenges of fracture risk assessment in primary care. Cleve Clin J Med 2021; 88(11):615-622. doi:10.3949/ccjm.88a.20199. On page 621, the second paragraph in the section titled "Pharmaceutical management recommended" should have read as follows: "Further, advising only the use of calcium and vitamin D is inadequate management. Her provider should recommend that she use an antiresorption agent as first-line therapy and consider anabolic drugs if there are problems with the initial drug choice. She should not reinstate hormone therapy at her age for bone health alone as there may be increased risk for cardiovascular disease. ${ }^{44}$ However, this caveat is not absolute and requires a balance of risk and reward if hormone therapy is also needed for vasomotor, genitourinary, or other problems." Reference 44 has been changed to the following: Flores VA, Pal L, Manson JE. Recommended hormone therapy in menopause: concepts, controversies and approach to treatment [published online ahead of print, 2021 Apr 15]. Endocr Rev 2021; bnab011. doi:10.1210/endrev/bnab011.

Article is correct on ccjm.org. 